\title{
Almost free actions on manifolds
}

\section{Philip T. Church and Klaus Lamotke}

Let $X$ be a compact, connected, oriented topological $G$-manifold, where $G$ is a compact connected lie group. Assume that the fixed point set is finite but nonempty, the action is otherwise free, and the orbit space is a manifold. It follows that either $G=U(1)=S^{1}$ and $\operatorname{dim} X=4$ or $G=S_{p}(1)=S^{3}$ and $\operatorname{dim} X=8$, and the number of fixed points is even. The authors prove that these $U(1)$-manifolds (respectively, $S_{p}(1)$-manifolds) are classified up to orientation-preserving equivariant homeomorphism by

(1) the orientation-preserving homeomorphism type of their orbit 3-manifolds (respectively, 5-manifolds), and

(2) the (even) number of fixed points.

Both the homeomorphism type in (1) and the even number in (2) are arbitrary, and all the examples are constructed. The smooth analog for $U(1)$ is also proved.

\section{Introduction}

In this paper we consider both the topological and the smooth $\left(C^{\infty}\right)$ categories. Manifolds are always assumed to be oriented, closed and connected, and homeomorphisms and diffeomorphisms are assumed to be orientation-preserving.

1.1. Let $X$ be a $G$-manifold, where $G$ is a compact connected Lie group, let $F \subset X$ be its fixed point set, let $X / G=N$ be its orbit space and let $\pi: X+N$ be the canonical map. Assume that $F$ is finite but

Received 24 October 1973. 
nonempty and the action is otherwise free. Call such an action almost free. Much of the information in $1.2-1.4$ is readily derived or even essentially known.

1.2. In the smooth case, $G=U(1)=S^{1}$ or $G=S U(2)=S_{p}(1)=S^{3}$. Furthermore, $N$ is a (topological) manifold if $G=S^{l}$ and $\operatorname{dim} X=4$ or $G=S^{3}$ and $\operatorname{dim} X=8$. In this case, $N$ has a canonical smooth structure.

1.3. In the topological case, if $N$ is a manifold, then $G=S^{1}$ and $\operatorname{dim} X=4$ or $G=S^{3}$ and $\operatorname{dim} X=8$.

1.4. The number of fixed points $\nu(F)$ equals the Euler characteristic $x(X)$.

1.5. If $N$ is a manifold, then $\nu(F)$ is even.

The main result of this paper is the following.

CLASSIFICATION THEOREM 1.6. Let $X$ and $X^{\prime}$ be almost free G-manifolds with $N$ and $N^{\prime}$ manifords.

(a) If $X$ and $X^{\prime}$ are equivariantly homeomorphic (diffeomorphic), then $N$ and $N^{\prime}$ are homeomorphic (diffeomorphic) and the number of fixed points $\nu(F)=\nu\left(F^{\prime}\right)$.

(b) Conversely, assume $N$ and $N^{\prime}$ are homeomorphic (diffeomorphic) and $v(F)=v\left(F^{\prime}\right)$. Then $X$ and $X^{\prime}$ are equivariantly homeomorphic. In the smooth case for $G=S^{1}, X$ and $X^{\prime}$ are equivariantly diffeomorphic. In the smooth case for $G=S^{3}$, the equivariant homeomorphism between $X$ and $X^{\prime}$ maps $X-F$ diffeomorphically onto $X^{\prime}-F^{\prime}$.

(c) The orbit manifold and number of fixed points can be arbitrary subject to $1.2,1.3,1.5$.

Theorem 1.6 remains true if "manifold" is reinterpreted to mean orientable manifold and a "homeomorphism (diffeomorphism)" is not assumed to be orientation-preserving. The authors do not know whether the result of $(b)$ in the smooth case for $G=S^{3}$ can be improved to: $X$ and $X^{\prime}$ are equivariantly diffeomorphic; see 2.10 and 4.6 below. 
This theorem extends Antonelli's [3] result on the classification of the space $X$ (but not the action) up to oriented homotopy type in case $N$ is a sphere and the action is smooth; see the remark in 4.3 below. The authors wish to acknowledge that the topic of this paper was suggested to them by Antonelli's paper.

This paper is organized as follows: in $\$ 2$ the action of $G$ on $X$ is studied locally near a fixed point. Especially, Proposition 2.3 and 2.5 yield 1.2 and Theorem $1.6(a)$, Proposition 2.6 yields 1.3 , and 2.7 yields 1.4. If the fixed points are removed, $X-F$ is a principal $G$-bundle over $N-\pi(F)$. Its characteristic (co)-homology class is studied in $\S 3$ : Proposition 3.5 (b) yields 1.5 and Proposition 3.8 yields Theorem 1.6 (b). In $\S 4$ sufficiently many examples of almost free $G$-manifolds are constructed in order to yield Theorem 1.6 (c).

\section{Local behaviour}

The action of $G$ on $X$ is studied locally in the neighborhood of a fixed point. Representation theory is the main tool in the smooth case, 2.1, Propositions 2.3 and 2.4, and 2.5, because local coordinates can be introduced such that $G$ acts orthogonally with respect to them.

2.1. Standard representations. Let $A$ denote the complex numbers $C$ or the quaternions $H$ and let $S^{p}=\{g \in A:|g|=1\}, p=1$ for $C$ and $p=3$ for $H$. Let $S^{p}$ act on $A \times A$ by

$$
g \cdot(z, w)=(g z, g w),
$$

respectively

$$
g \cdot(z, w)=(z \bar{g}, g w) .
$$

This action is almost free, that is, free except for the fixed point $(0,0)$, the orbit space is $A \times A$ and the projection map

$$
(z, w)=(2 \bar{z} \omega, w \bar{w}-z \bar{z}) \text {, }
$$

respectively

$$
(z, w)=(2 z w, w \bar{w}-z \bar{z}),
$$

compare [14, p. 102]. Restrictions of (1a) and (1b) yield actions of $S^{p}$ on the unit sphere $S^{2 p+1}$ and the unit disk $D^{2 p+2}$ in $A \times A$ with orbit 
spaces the unit sphere $S^{p+1}$ and the unit disk $D^{p+2}$ in $A \times R$ respectively. These $S^{p}$-manifolds are denoted by $\pm S^{2 p+1}$ and $\pm D^{2 p+2},+$ for ( $1 a$ ) and - for (1b). The bundles $\gamma_{ \pm}$with total space $\pm S^{2 p+1}$ and projection given by (2a), respectively $(2 b)$, are the $( \pm)$ Hopf bundles. The orientation reversing equivariant diffeomorphism given by $\psi(z, w)=(\bar{z}, w)$ transforms (la) into (lb). We call these representations standard because of (d) of the following proposition.

PROPOSITION 2.2. (a) The only compact connected Lie groups $G$ having almost free (that is, free except for the origin) real representations are $U(1)=S^{1}$ and $S U(2)=S p(1)=S^{3}$.

(b) The usual representation of $U(1)$ on $C$ and of $S U(2)$ on $H$ is almost free.

(c) Up to equivalence every real almost free representation is a direct sum of several copies of this representation.

(d) The orbit space is a manifold if and only if the number of copies is two, that is, up to orientation preserving equivalence the representation is the one described in 2.1.

Proof. (a) Since $G$ is compact, the representation can be assumed to be orthogonal. Therefore the unit sphere $S$ in the representation space is invariant and $G$ acts freely on $S$. Thus $S$ is the total space of a bundle with fibre $G$ and it follows from [5] that $G$ has the homotopy type of $S^{1}, S^{3}$, or $S^{7}$. Since $G$ is a Lie group, it is $S^{1}=U(1)$ or $S^{3}=S U(2)$.

Conclusion (b) is trivial.

(c) Let $A$ denote the usual complex representation of $U(1)$ on $C$, respectively of $S U(2)$ on $C^{2}$, and let $A_{p}$ denote $A$ considered as real representation. Then $A_{r}$ and hence $A$ are irreducible. From the explicit description of all irreducible complex representations of $U(I)$ (see [1, pp. 77-78]) and of $S U(2)$ (see [16, p. 58]), it follows that none of them is almost free except $A$. Let $B$ be some almost free not necessarily irreducible real representation. Then the complex 
representation $B \otimes C$ is almost free. For $B \otimes C=B \oplus B$ as real representations, and the direct sum of representations is almost free if and only if each summand is almost free. The same reason implies: $B \otimes C=A \oplus \ldots \oplus A \quad$ ( $m$ terms $)$ as complex representations and hence $B \oplus B=A_{\boldsymbol{r}} \oplus \ldots \oplus A_{\boldsymbol{r}} \quad$ (m terms) as real representations. Since the decomposition of a representation into irreducible components is unique, $B=A_{r} \oplus \ldots \oplus A_{r} \quad(k$ terms, where $2 k=m)$.

(d) Assume "two copies": the orbit space is a manifold according to 2.1. Assume "manifold": let $D_{n}$ denote the unit disk in the representation space. Then $D^{n} / G$ is the cone over $S^{n-1} / G$ because the action is orthogonal. Since $D^{n} / G$ is assumed to be a manifold, $S^{n-1} / G$ must be a sphere. On the other hand, $s^{n-1} / G$ is the base space of the fibre bundle with total space $S^{n-1}$ and fibre $G=S^{1}$, respectively $=S^{3}$ (compare (a) of the proof). Then $n=4$ for $G=S^{1}$, respectively $n=8$ for $G=S^{3}$, is a consequence of the homotopy sequence of a fibering.

PROPOSITION 2.3. Let $X$ be a smooth almost free G-manifold. Then

(a) the group is $G=S^{1}$ or $=s^{3}$,

(b) the orbit space is a smooth manifold if and only if $G=S^{1}$ and $\operatorname{dim} X=4$ or $G=S^{3}$ and $\operatorname{dim} X=8$. In this case the formulas ( $1 \mathrm{a})$, respectively ( $1 \mathrm{~b})$, and (2a), respectively $(2 \mathrm{~b})$, describe the action and projection with respect to local coordinates near any fixed point.

Proof. The slice theorem, see, for example, [12, p. 3], implies: in some neighborhood of a fixed point there are smooth coordinates sich that $G$ acts linearly. Thus Proposition 2.3 is a consequence of Proposition 2.2 except for the smooth structure of $N$ which will be dealt with in 2.5 below.

2.4. The associated bundle (topological and smooth case). Since $G$ acts freely on $X-F$, the restriction $\pi: X-F \rightarrow N-\pi(F)$ is the projection of a (smooth) locally trivial principal $G$-bundle $\xi$, see [4, 
p. 157], for the topological and [12, p. 8], for the smooth case. Since $G$ and $X-F$ are oriented, $N-\pi(F)$ is oriented, and so is $N$, if $N$ is a manifold: precisely, first $G$ then $N-\pi(F)$ gives the orientation of $X-F$.

2.5. The smooth structure of the orbit space. Let $X$ be a smooth almost free $G$-manifold, $G=S^{1}$ and $\operatorname{dim} X=4$ or $G=S^{3}$ and $\operatorname{dim} X=8$. Being the base space of a smooth bundle, $N-\pi(F)$ is a smooth manifold. The local coordinate description given in 2.1 determines a smooth structure in the neighborhood $U$ of the image $\pi(x)$ of each fixed point $x$. The smooth structures in $N-\pi(F)$ and $U$ are compatible: with respect to either structure, for every open subset $V \subset U-\pi(x)$, a function $f: V \rightarrow R$ is smooth if and only if $f \circ \pi$ is smooth, because $\pi \mid X-F$ has maximal rank and so does $\pi$ given by formula (2a) or (2b) outside 0 .

The uniqueness of this smooth structure and the only non-trivial part of Theorem $1.6(a)$ are consequences of the following proposition.

PROPOSITION. Let $X$ and $X^{\prime}$ be as above, and let $\alpha: X \rightarrow X^{\prime}$ be an equivariant diffeomorphism. Then $N$ is diffeomorphic to $N^{\prime}$.

Proof. Let $\bar{\alpha}: N \approx N^{\prime}$ be the induced homeomorphism, and let $K$ be the union of disjoint closed $(p+2)$-disks, each containing precisely one point of $\pi(F)$. Then $\bar{\alpha}$ maps $N$ - int $K$ diffeomorphically onto $N^{\prime}$ - int $\bar{\alpha}(K)$. Since any diffeomorphism of $S^{p+1}$ can be extended to a diffeomorphism of $D^{p+2}$ for $p=1$ or $=3$ (that is, $\Gamma_{3}=\Gamma_{5}=0 \quad[7$, p. ix]), $\bar{\alpha}$ restricted to $N$ - intK can be extended to a diffeomorphism of $N$ onto $N^{\prime}$.

PROPOSITION 2.6 (topological case). Let $X$ be an almost free G-manifold with $N=X / G$ a manifold. Then

$$
G=S^{1}, \operatorname{dim} X=4 \text {, and } \operatorname{dim} N=3
$$

or

$$
G=S^{3}, \operatorname{dim} X=8, \text { and } \operatorname{dim} N=5 .
$$

Let $p=1$ or $=3$. For any $a \in F$ and bicolzared embedding $j:\left(D^{p+2}, 0\right) \rightarrow(N, \pi(a))$ such that $j\left(D^{p+2}\right) \cap \pi(F)=\pi(a)$, there is an 
equivariant embedding $\lambda: \pm D^{2 p+2} \rightarrow X$, see 2.1, with $\lambda(0)=a$ and $\pi \lambda\left(D^{2 p+2}\right)=j\left(D^{p+2}\right)$. The point a (and $\left.\pi(a)\right)$ is said to have index $d_{a}= \pm 1$ according as $\pm D^{2 p+2}$ is required, and the index is independent of the choice of $j$.

Proof. The map $\pi: X \rightarrow X / G$ is a singular fibering [8] and it follows from [20] that $\pi$ restricted to $\pi^{-1}\left(j\left(D^{q}\right)\right)$ is topologically equivalent to the cone map of a fibre bundle with total space $S^{n-1}$, base space $s^{q-1}$, and fibre a homotopy $(n-q)$-sphere,

$$
(n, q)=(4,3),(8,5) \text {, or }(16,9) \text {. }
$$

Since this is a principal $G$-bundle $\xi, 2.4$, where $G$ is a lie group, $G=S^{l}$ or $=S^{3}$. We now prove that the only principal $S^{p}$-bundle over $s^{p+1}$ with total space a homotopy $S^{2 p+1}$ is the $( \pm)$ Hopf bundle. Since the Hopf bundle $\gamma_{+}$is $(2 p+1)$-universal, every principal $S^{p}$-bundle over $S^{p+1}$ is induced from $\gamma_{+}$by a map $f: S^{p+1}+S^{p+1}$. Let $E$ be the total space of $f * \gamma_{+}$and $m=\mid$ degree $f \mid$. Comparison of the exact homotopy sequences of $f * \gamma_{+}$and $\gamma_{+}$yields $\pi_{p}(E)=Z_{m}$. Hence $E \simeq S^{2 p+1}$ implies $m=1$. Since $f * \gamma_{+}$depends only on the homotopy class of $f, f * \gamma_{+}=\gamma_{ \pm}$. The homotopy class of the restriction $j \mid S^{p+1}: S^{p+1} \rightarrow N-\pi(a)$ is uniquely determined by $a$. Hence the equivalence class of $\left(j \mid S^{p+1}\right) * \xi$, that is, the sign of the Hopf bundle, does not depend on the choice of $j$.

2.7. Proof of 1.4. The Lefschetz fixed point theorem is used, see [9]. Choose some element $g \in G, g \neq 1$. Then $g: X \rightarrow X$ is an homeomorphism with fixed point set $F$. Since $G$ is connected, $g$ is homotopic to the identity. Therefore $x(X)=\Sigma_{\lambda} I\left(g, q_{\lambda}\right)$, where $F=\left\{q_{1}, \ldots, q_{1}\right\}$, and $I\left(g, q_{\lambda}\right) \in 2$ denotes the fixed point index of $g$ at $q_{\lambda}$. Hexe $I\left(g, q_{\lambda}\right)$ depends only on $g$ restricted to some neighborhood $V$ of $q_{\lambda}$, more precisely: choose coordinates in $V$; so that the 
restriction of $g$ can be considered as a map $g: V \rightarrow R^{n}$. The index $I(g, q)$ is defined by $(i d-g)_{*}: H_{n}\left(V, V-q_{\lambda}\right) \rightarrow H_{n}\left(R^{n}, R^{n}-0\right)$, $\mathrm{o}_{1} \mapsto I\left(g, q_{\lambda}\right) \cdot \mathrm{o}_{2}$. Here $i d-g:\left(V, V-q_{\lambda}\right) \rightarrow\left(R^{n}, R^{n}-0\right)$, $x \mapsto x-g x \cdot H_{n}\left(V, V-q_{\lambda}\right) \approx z$ and $H_{n}\left(R^{n}, R^{n}-0\right) \approx z$ are the homology groups with integer coefficients, and $0_{1}, 0_{2}$ denote generating elements which are determined by means of the same orientation of $R^{n}$. In our case, use the local coordinate description of $g$ given by the formulas (1a), respectively (1b), in 2.1. We conclude: $i d-g$ is an orientation preserving homeomorphism; hence $I\left(g, q_{\lambda}\right)=1$ for every $\lambda$ and thus $\chi(X)=\Sigma_{\lambda} I\left(g, q_{\lambda}\right)=\nu(F)$.

2.8. Let $D^{2 p+2}$ and $2 D^{2 p+2}=S^{2 p+1}$ be the $S^{p}$-manifolds of 2.1 with orbit spaces $D^{p+2}$ and $\partial D^{p+2}=S^{p+1}$, respectively, $p=1$ or 3 . The following problem will be studied. Given an equivariant homeomorphism, respectively diffeomorphism, $\alpha$ of $S^{2 p+1}$ onto itself, can it be extended to an (equivariant ?) homeomorphism, respectively diffeomorphism, $\alpha$ of $D^{2 p+2}$ ? The answer is yes in the topological case. Define $\beta: D^{2 p+2} \approx D^{2 p+2}, t x+t \alpha(x)$ for $x \in s^{2 p+1}$ and $0 \leq t \leq 1$. But for a diffeomorphism $\alpha$, this $\beta$ need not be smooth at the origin.

The following proposition partially answers the question in the smooth case. This result will be used in order to prove Theorem 1.6 (b).

PROPOSITION. Given an equivariant diffeomorphism $\alpha$ of $D^{4}-0$ onto itself, there is an equivariant diffeomorphism $B$ of $D^{4}$ onto itself such that $\beta=\alpha$ in some neighborhood of $\partial D^{4}$.

The following conventions will be used.

$I=[0,1]$ is the unit interval. The $S^{p}$-action on $S^{2 p+1} \times I$ is defined by $g \cdot(x, t)=(g x, t)$.

From the (equivariant for $n=2 p+2$ and $p=1$ or $=3$ ) diffeomorphism $s^{n-1} \times(0,1] \approx D^{n}-0,(x, t) \rightarrow t x$, subsets of 
$S^{n-1} \times(0,1]$ will be identified with subsets of $D^{n}-0$.

For a map $f$ between $S^{P}$-manifolds, the induced map of orbit spaces is denoted by $f$.

LEMMA 2.9. Under the hypothesis of the proposition there is an equivariant diffeomorphism $\gamma$ of $S^{2 p+1} \times(0,1]$ onto itself such that $\gamma=\alpha$ on a neighborhood of $S^{2 p+1} \times 1 \approx \partial D^{2 p+2}$ and $\bar{\gamma}=$ id on $s^{p+1} \times(0,2 / 3]$.

Proof. The diffeomorphism $\bar{\alpha} \mid S^{p+1}$ can be extended to a diffeomorphism of $D^{p+2}$ onto itself $\left(\Gamma_{3}=\Gamma_{5}=0,[9], p\right.$. ix $)$, and using [15, p. 551], this extension can be chosen in such a way as to yield a diffeomorphism $f$ of $s^{p+1} \times(0,1]$ onto itself such that $f(u, t)=(u, t)$ for $0 \leq t \leq 2 / 3$ and $f(u, t)=\bar{\alpha}(t u)$ on a neighborhood of $s^{2 p+1} \times 1$. Now $S^{2 p+1}$ is a fibre bundle over $s^{p+1}$ and by the covering homotopy theorem, [19, p. 50], in the smooth version there is an equivariant diffeomorphism $\gamma$ of $S^{2 p+1} \times(0,1]$ onto itself such that $\gamma=\alpha$ on a neighborhood of $s^{2 p+1} \times 1$ and $\bar{\gamma}=f$.

2.10. Proof of 2.8. Here $p=1$. Let $\gamma$ be as given by Lemma 2.9. Since $\bar{\gamma}=i d$ for $(x, t)$ with $0<t \leq 2 / 3$, and since $S^{l}$ is abelian, there is a smooth $\rho: S^{2} \times(0,2 / 3] \rightarrow S^{1}$ such that $\gamma(x, t)=(\rho(\pi(x), t) \cdot x, t)$ for $0<t \leq 2 / 3$. Since the second homotopy group $\pi_{2}\left(s^{l}\right)=0$ there is a smooth $\sigma: s^{2} \times(0,2 / 3]+s^{1}$ such that $\sigma(y, t)=\rho(y, t)$ for $t$ in a neighborhood of $2 / 3$ and $\sigma(y, t)=1$ for $0<t \leq 1 / 2$. Define $B(t x)=\gamma(x, t)$ for $2 / 3 \leq t \leq 1$, $B(t x)=(\sigma(\pi(x), t) \cdot x, t)$ for $1 / 2 \leq t \leq 2 / 3$, and $B(t x)=t x$ for $0 \leq t \leq 1 / 2$.

REMARK. Since $S^{3}$ is non-abelian, and since the relevant homotopy group $\pi_{4}\left(S^{3}\right)=z_{2} \neq 0$, the argument given above cannot be extended to the case $p=3$. 


\section{The characteristic class}

In 2.4 a bundle $\xi$ has been associated with the almost free $G-$ manifold $X$. The global behaviour of the action of $G$ on $X$ will be studied by means of the characteristic homology class $c(\xi)$ of this bundle. Singular homology and cohomology with integer coefficients are consistently used.

3.1. Classification of principal $S^{p}$-bundles, $p=1$ or $=3$. Let $n$ be an universal principal $S^{P}$-bundle with base space (= classifying space) $B$. Let $l \in H^{p+1}(B) \approx Z$ denote the fundamental class. Then for any paracompact topological space $U$ having the homotopy type of a CW-complex

$$
\alpha:[U, B]+E(U), f \rightarrow f^{*} \eta,
$$

is an isomorphism [11]. Here $[U, B]$ denotes the set of homotopy classes of maps $U \rightarrow B$ and $E(U)$ denotes the set of isomorphism classes of principal $S$-bundles over $U$. On the other hand, define

$$
B:[U, B] \rightarrow H^{p+1}(U), f \rightarrow f^{*}(\imath) .
$$

The composition

$$
\bar{c}: E(U) \frac{\alpha^{-1}}{\tilde{\imath}}[U, B] \stackrel{B}{\longrightarrow} H^{p+1}(U)
$$

is the "characteristic class". If $p=1, \bar{c}$ is the first Chern class. For the Hopf bundle $\gamma^{p}$ over $s^{p+1}, c\left(\gamma^{p}\right)$ is a generator of $H^{p+1}\left(S^{p+1}\right) \approx z$. If $H^{q}(U, M)=0, q>p+1$, for all coefficient modules $M, \beta$ and hence $\bar{c}$ is an isomorphism [18, p. 447, Theorem 3].

REMARK 3.2. Let $N$, be a $(p+2)$-dimensional manifold, and let $A \neq \emptyset$ be a closed subset of $N$. Then $U=N-A$ satisfies all hypotheses of 3.1 , so that the principal $S^{p}$-bundles $\xi$ over $N-A$ are classified by their characteristic classes $\bar{c}(\xi) \in H^{p+1}(N-A)$.

3.3. Let $X$ be an almost free $S^{p}$-manifold $(p=1$ or $=3)$ with fixed point set $F$ and orbit marifold $N=X / S^{p}$. According to 
Proposition 2.6, $\operatorname{dim} N=p+2$. Therefore 3.1 and Remark 3.2 apply. The associated bundle $\xi$ as defined in 2.4 is characterized by $\bar{c}(\xi) \in H^{p+1}(N-\pi(F)) ;$ more precisely:

LEMMA. Let $X$ and $X^{\prime}$ be two almost free $S^{p}$-manifolds with fixed point sets $F$ and $F^{\prime}$, orbit manifolds $N$ and $N^{\prime}$ and associated bundles $\xi$ and $\xi^{\prime}$. If there is a homeomorphism $f: N \rightarrow N^{\prime}$ such that

$$
f \pi(F)=\pi^{\prime}\left(F^{\prime}\right)
$$

and

$$
\bar{c}(\xi)=f^{*} \bar{c}\left(\xi^{\prime}\right),
$$

then $X$ and $X^{\prime}$ are equivariantly homeomorphic. In the smooth case, if $f$ is a diffeomorphism, then $X$ and $X^{\prime}$ are equivariantly diffeomorphic in the case $p=1$. In the case $p=3$ there is an equivariant homeomorphism $\mu: X \rightarrow X^{\prime}$ such that $\mu$ maps $X-F$ diffeomorphically onto $X^{\prime}-E^{\prime}$.

Proof. Given $f$ satisfying (1) and (2), then 3.1 implies: the bundle $\xi$ is equivalent to the induced bundle $f^{*} \xi^{\prime}$. Thus there is an equivariant homeomorphism (diffeomorphism) $\mu: X-F \rightarrow X^{\prime}-F^{\prime}$, so that the induced map of the orbit spaces is $f$. By filling in the fixed points $F$, respectively $F^{\prime}, \mu$ is extended to an equivariant homeomorphism $\mu: X \rightarrow X^{\prime}$. But in the smooth case, this $\mu$ need not be smooth at the fixed points. For $p=1$ it will be smoothed in the following way. In the neighborhood of every fixed point in $X$ or $X^{\prime}$ smooth coordinates are chosen so that the $S^{l}$-action is given by 2.1 ; in other words for every fixed point $x_{v} \in X$ and its image $x_{v}^{\prime}=\mu\left(x_{v}\right) \in X^{\prime}$ there are smoothly and equivariantly embedded disks $\left(D_{v}^{4}, 0\right) \subset\left(x, x_{v}\right)$ and $D_{\nu}^{\prime 4} \subset\left(X^{\prime}, x_{\nu}^{\prime}\right)$ such that the restriction of $\mu$ is an equivariant diffeomorphism $D_{v}^{4}-0 \approx D_{v}^{\prime 4}-0$. The induced map $D_{v}^{3}-0 \approx D_{v}^{\prime 3}-0$ of the orbit spaces is the restriction of $f$, hence may be extended to a diffeomorphism $D_{\nu}^{4} \approx D_{v}^{\prime 4}$. Thus 2.8 applies: there is an equivariant diffeomorphism $\mu_{v}: D_{v}^{4} \approx D_{v}^{\prime 4}$ such that $\mu_{v}=\mu$ in a neighborhood of 
$\partial D_{\nu}^{4}$. Then $\mu^{\prime}: X \approx X^{\prime}$ with $\mu^{\prime}=\mu$ off $\bigcup_{\nu} D_{\nu}^{4}$ and $\mu^{\prime}=\mu_{\nu}$ in $D_{\nu}^{4}$ is an equivariant diffeomorphism.

3.4. It will be more convenient to replace the cohomology class $\bar{c}(\xi)$ by its dual homology class $c(\xi)$, since $c(\xi)$ is represented by a onedimensional chain, that is, by a rather simple geometric object. The definition of $e(\xi)$ runs as follows. Let $q_{\lambda}(\lambda=1,2, \ldots, 2)$ be the points of $\pi(F)$, let $D_{\lambda}$ be disjoint $(p+2)$-cells in $N=N^{p+2}$ with $q_{\lambda} \epsilon \operatorname{int} D ;$, and let $E=U_{\lambda}^{U} \operatorname{int} D_{\lambda}$. Let $\varphi$ be the composition isomorphism

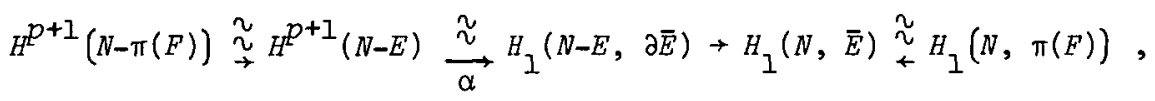

where $\alpha$ is the duality isomorphism, [19, p. 305], and the other isomorphisms are induced by inclusion. Define

$$
c(\xi)=\varphi \bar{c}(\xi) \text {. }
$$

Then 3.3 (2) is equivalent, [18, p. 254], to

$$
c\left(\xi^{\prime}\right)=f_{*} c(\xi) \text {. }
$$

PROPOSITION 3.5. (a) For the connecting homomorphism $\partial: H_{1}(N, \pi(F)) \rightarrow H_{0}(\pi(F))$, the $\lambda$ th component of $\partial c(\xi)$ is the index $d_{\lambda}\left(= \pm 1\right.$ ) of $q_{\lambda}$, (Proposition 2.6).

(b) The number of fixed points is even: $v(F)=2=2 k$ $(k=1,2, \ldots)$, and after reordering $d_{\lambda}=(-1)^{\lambda}$.

(b) yields 1.5 .

Proof. (a) For $j: D \rightarrow N$ as in Proposition 2.6, $\left(j \mid S^{p+1}\right)^{*} \bar{c}(\xi)=d_{a}\left[S^{p+1}\right]$, where $\left[S^{p+1}\right]$ is the fundamental cohomology class and $d_{\alpha}$ is the index $( \pm 1)$ of $a$. This fact and the commutativity of the diagram below imply $(a)$ of the proposition.

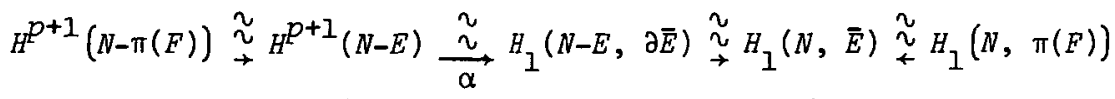

$$
\begin{aligned}
& H^{p+1}(\partial \bar{E}) \underset{\beta}{\rightarrow} H_{0}(\partial \bar{E}) \stackrel{\approx}{\sim} H_{0}(E) \stackrel{\approx}{\longleftarrow} H_{0}(\pi(E)) .
\end{aligned}
$$


Here the top row is the isomorphism $\varphi, 3.4, \alpha$ and $\beta$ are duality isomorphisms, the $\partial^{\prime} \mathrm{s}$ denote connecting homomorphisms, the remaining homomorphisms are induced by inclusions. The commutativity of the square containing $\alpha$ and $\beta$ can be found in [18, p. 255]. The other partial diagrams are trivially commutative.

(b) Let $i: \pi(\Gamma) \rightarrow N$ be the inclusion. Then from $(a)$, $i_{*} \partial c(\xi)=\Sigma_{\lambda} d_{\lambda}$ (here $H_{0}(N)$ is identified with $Z$ ). From the exactness of the homology sequence of $(N, \pi(F)), i_{*} \partial=0$, that is, $\Sigma_{\lambda} d_{\lambda}=0$. Since $d_{\lambda}= \pm 1$, there must be as many $d_{\lambda}=+1$ as $d_{\lambda}=-1$. (b) of the proposition follows.

REMARK. If $z_{2}$ coefficients are used, the argument yields 1.5 in case $N$ (and thus $X$ ) is non-orientable.

The following section, Lemmas 3.6 and 3.7 , and Proposition 3.8, prepare the proof, 3.9 , of Theorem 1.6 (b).

LEMMA 3.6. Given a path $\gamma:[0,1] \rightarrow N^{p+2}, N$ a (smooth) manifold and $p \geq 0$, given $0=t_{1}<t_{2}<\ldots<t_{l}=1$ with $\gamma\left(t_{\lambda}\right)$ distinct, there are a path $\delta$ homotopic to $\gamma$ with the $t_{\lambda}$ fixed and a bicollared (smooth) closed $(p+2)-c e l l \quad D \subset N$ with imaged $\subset \operatorname{int} D$.

Proof. We may suppose that $\gamma(t) \neq\left(t_{\lambda}\right)$ for every $t \neq t_{\lambda}$. Let $J=\{s \in[0,1]:$ there are a path $\alpha$ and a bicollared (smooth) closed $(p+2)-\operatorname{cell} E \subset N$ with $\alpha([0, s]) \subset \operatorname{int} E$ and $\alpha$ homotopic to $\gamma$ with $t_{\lambda}$ and all $t \geq s$ fixed .

Then $0 \in J, J$ is open, and it suffices to prove that $J$ is closed: let $s_{1}$ be such that every open interval containing $s_{1}$ meets $J$. We shall prove $s_{I} \in J$. Let $U \subset N$ be a closed $(p+2)$-cell with $\gamma\left(s_{1}\right) \in$ int $U$ and $\gamma\left(t_{\lambda}\right) \notin U$ unless $s_{1}=t_{\lambda}$. There is $s_{0} s_{1}$ with $\gamma\left(\left[s_{0}, s_{1}\right]\right) \subset \operatorname{int} U$ and $s_{0} \in J$. Then $\alpha$ and $E$ are given for $s=s_{0}$. Let $D_{\rho}=\left\{x: x \in R^{p+2},\|x\| \leq \rho\right\}$ denote the disk of radius $\rho$. There is a homeomorphism (diffeomorphism) $\mu: D_{1} \approx U$ with $\mu(0)=\gamma\left(s_{0}\right)$, 
$\mu\left(D_{1 / 3}\right) \subset E$, and $\gamma\left(\left[s_{0}, s_{1}\right]\right) \subset \mu\left(D_{2 / 3}\right)$. Thus there is a (smooth) isotopy $H_{t}: N \approx N$ such that $H_{t} \mid(N-\operatorname{int} U)$ is the identity and $H_{t}(\mu(0))=\mu(0), H_{0}$ is the identity, and $H_{1}\left(\mu\left(D_{1 / 3}\right)\right)=\mu\left(D_{2 / 3}\right)$. Let $E^{\prime}=H_{1}(E)$ and let $\alpha^{\prime}(t)=H_{1}(\alpha(t))$ for $t \leq s_{0}$ and $=\gamma(t)$ otherwise. Since $\alpha\left(\left[s_{0}, s_{1}\right]\right) \subset \mu\left(D_{2 / 3}\right)=H_{1}\left(\mu\left(D_{1 / 3}\right)\right) \subset H_{1}(E)=E^{\prime}$, it follows that $s_{1} \in J$.

REMARK. In the smooth case, a much simpler argument suffices. There is a piecewise linear arc $\delta$ homotopic to $\gamma$ with the $t_{\lambda}$ fixed and we may simply choose $D$ to be a smooth regular neighborhood of image $\delta$.

LEMMA 3.7. There are a bicolzared (smooth) (p+2)-cell $j: D \subset N$ with $\pi(F) \subset \operatorname{int} D$, and $b \in H_{1}(D, \pi(F))$ with $j_{\star} b=c(\xi)$ (notation as in 3.4).

Proof. Let $E=\left\{q_{1}, \ldots, q_{2 k}\right\}$, let $\alpha_{\lambda}(\lambda=1,2, \ldots, 2 k-1)$ be a path joining $q_{\lambda}$ to $q_{\lambda+1}$, and let $\operatorname{cl} \alpha_{\lambda} \in H_{1}(N, \pi(F))$ be its homology class. Then by Proposition 3.5, $\sum_{\lambda \text { odd }} c l \alpha_{\lambda}=\partial c(\xi)$, and from the exactness of the homology sequence there is an $a \in H_{1}(N)$ such that $i_{*} a=c(\xi)-\sum_{\lambda \text { odd }} c l \alpha_{\lambda}$. Let $\beta$ be a loop at $q_{1}$ such that $\operatorname{cl} \beta=a$, let $\gamma_{1}=\beta \alpha_{1}$ (product of paths), and let $\gamma_{\lambda}=\alpha_{\lambda}$ for $\lambda>1$. Then $\lambda$ odd $c \perp \gamma_{\lambda}=c(\xi)$. Let $D$ and $\delta$ be as given by Lemma 3.6 for $\gamma=\gamma_{1} \gamma_{2} \ldots \gamma_{2 k-1}$ and $t_{\lambda}=(\lambda-1) /(2 k-1)$. Define $\delta_{\lambda}(t)=t t_{\lambda}+(1-t) t_{\lambda-1}, 0 \leq t \leq 1$, and $b=\sum_{\lambda \text { odd }} c 1 \delta_{\lambda} \in H_{1}(D, \pi(F))$.

PROPOSITION 3.8. Let $M$ be an orientied, connected n-dimensional manifold without boundary, $n \neq 1, n \neq 4$ ( $n \neq 1$ only, in the smooth case). Let $D_{1}^{n}, D_{2}^{n} \subset M \cdot$ be two bicolzared (smooth) disks. Let $p_{\lambda} \in \operatorname{int} D_{1}, q_{\lambda} \in \operatorname{int} D_{2}, \lambda=1, \ldots, \tau, p_{\lambda} \neq p_{\mu}$ and $q_{\lambda} \neq q_{\mu}$ for $\lambda \neq \mu$, be finitely many points. Then there is a homeomorphism 
(diffeomorphism) $f: M \approx M$ such that $f\left(D_{1}\right)=D_{2}$ and $f\left(p_{\lambda}\right)=q_{\lambda}$ for $\lambda=1, \ldots, l$.

For the proof of this proposition combine the following facts.

(a) Let $p_{1}, \ldots, p_{\eta}, q_{1}, \ldots, q_{l} \in \operatorname{int} D^{n}$ with $p_{\lambda} \neq p_{\mu}$ and $q_{\lambda} \neq q_{\mu}$ for $\lambda \neq \mu$ be finitely many points. There is a diffeomorphism $h: D^{n} \approx D^{n}$ such that $h\left(p_{\lambda}\right)=q_{\lambda}, \lambda=1, \ldots, l$ and $h=i d$ in the neighborhood of the boundary $s^{n-1}$.

(b) Homogeneity of manifolds in the smooth case, [17]. Given two embeddings $j_{1}, j_{2}: D^{n} \rightarrow M^{n}$, there is a diffeomorphism $f: M^{n} \rightarrow M^{n}$ with $j_{2}=f \cdot j_{1} \cdot$

(c) Homogeneity of manifolds in the topological case. Given two bicollared disks $D_{1}^{n}, D_{2}^{n} \subset M^{n}$. There is an homeomorphism $f: M^{n} \rightarrow M^{n}$ such that $h\left(D_{1}\right)=D_{2}$. This is a consequence of the annulus conjecture. (Let $D_{1}^{n} \subset D_{2}^{n}$ be bicollared; then $d_{2}^{n}-\operatorname{int} D_{1}^{n}$ is homeomorphic to $s^{n-1} \times[0,1]$.) This has been proved for $n \leq 3,[6]$ and $n \geq 5,[13]$.

3.9. Proof of Theorem $1.6(b)$. Let $D, b$ in $N$ and $D^{\prime}, b^{\prime}$ in $N^{\prime}$ be chosen as in Lemma 3.7. Let $\pi(F)=\left\{q_{1}, \ldots, q_{2 k}\right\} \subset N$ and $\pi^{\prime}\left(E^{\prime}\right)=\left\{q_{1}^{\prime}, \ldots, q_{2 k}^{\prime}\right\} \subset N^{\prime}$ be the fixed points. Since $N \approx N^{\prime}$, Proposition 3.8 implies there is an $f: N \approx N^{\prime}$ with $f(D)=D^{\prime}$ and $f\left(q_{\lambda}\right)=q_{\lambda}^{\prime}$ for $\lambda=1, \ldots, 2 k$. From Proposition 3.5, $f_{*} \partial c(\xi)=\partial c\left(\xi^{\prime}\right)$; hence $\partial h_{*} b=\partial b^{\prime}$. Here $h: D \approx D^{\prime}$ denotes the restriction of $f$. Since $\partial$ on $H_{1}\left(d^{\prime}, \pi\left(F^{\prime}\right)\right)$ is a monomorphism, $h_{*} b=b^{\prime}$. Thus $f: N \approx N^{\prime}$ satisfies $f(\pi(E))=\pi^{\prime}\left(F^{\prime}\right)$ and $f_{*} c(\xi)=c\left(\xi^{\prime}\right)$ or $3.4(3)$, equivalently $f_{*} \bar{c}\left(\xi^{\prime}\right)=\bar{c}(\xi)$, and Theorem (b) results from 3.3 .

\section{Examples}

In this chapter sufficiently many almost free $S^{p}$-manifolds are described explicitly in order to prove Theorem 1.6 (c). The conventions concerning $A$ and $p=1$ or $=3$ are the same as in 2.1. 
4.1. The $S^{p}$-manifold $S^{2 p+2}$. Let $S^{p}$ act on $A^{2} \times R$ by $g \cdot(z, w, x)=(g z, g w, z)$. Then $S^{2 p+2}=\left\{(z, w, x): z \bar{z}+w \bar{\omega}+x^{2}=1\right\}$ is invariant and the operation of $S^{p}$ on $S^{2 p+2}$ is free except for the two fixed points $(0,0, \pm 1)$. The orbit space is

$$
s^{p+2}=\left\{(a, s, t) \in A \times R^{2}: a \bar{a}+s^{2}+t^{2}=1\right\},
$$

and the projection is $\pi(z, w, x)=\left(2 \bar{z} \omega, w \bar{\omega}-z \bar{z}, x\left(2-x^{2}\right)^{\frac{1}{2}}\right)$. Equivalently, identify the boundaries of $+D^{2 p+2}$ and $-D^{2 p+2}$ by the identity to form this $S^{p}$-manifold.

4.2. The $S^{p}$-manifold $S^{p+1} \times S^{p+1}$. Let $S^{p}$ act on $A^{2} \times R^{2}$ by $g(z, w, x, y)=(g z, g w, x, y)$. Then

$$
S^{p+1} \times S^{p+1}=\left\{(z, w, x, y): z \bar{z}+x^{2}=1, w \bar{w}+y^{2}=1\right\}
$$

is invariant, and the operation of $S^{p}$ on $s^{p+1} \times s^{p+1}$ is free except for the four fixed points $(0,0, \pm 1, \pm 1)$. The orbit space is $S^{p+2}$ as in 4.1 , and the projection is $\pi(z, w, x, y)=\left(1+x^{2} y^{2}\right)^{\frac{1}{2}}(\bar{z} \omega, x, y)$ (scalar multiplication).

4.3. The equivariant connected sum $\#$. Let $x_{j}(j=1,2)$ be two almost free $S^{p}$-manifolds with orbit manifolds $N_{j}$ and fixed point sets $F_{j}$; let $x_{j} \in X_{j}$ be two fixed points with index $\left(x_{1}\right)=+1$ and index $\left(x_{2}\right)=-1$, see Proposition 2.6. Let $\lambda_{j}:\left(+D^{2 p+2}, 0\right) \rightarrow\left(x_{j}, x_{j}\right)$ be equivariant (smooth) embeddings, $\lambda_{1}$ orientation preserving, $\lambda_{2}$ orientation reversing. Form the identification space from $X_{1}-x_{1}$ and $x_{2}-x_{2}$ by identifying $\lambda_{1}(t u)$ with $\lambda_{2}((1-t) u)$ for each $u \in S^{2 p+1}$, $0<t<1$. The resulting $S^{p}$-manifold is called the equivariant connected sum of $x_{1}$ and $x_{2}$, and is denoted by $x_{1} \# x_{2}$. Observe: disregarding the $S^{P}$-action, this is the usual connected sum; $X_{1} \# X_{2}$ is almost free; 
the orbit space $\left(X_{1} \# X_{2}\right) / S^{p}$ is the connected sum $N_{1} \# N_{2}$; the number of fixed points is $v\left(F\left(X_{1} \# X_{2}\right)\right)=v\left(F_{1}\right)+v\left(F_{2}\right)-2$. Up to equivariant homeomorphism in the topological $S^{l}$ and $S^{3}$ case, up to equivariant diffeomorphism in the smooth $S^{l}$ case and up to (possibly non-equivariant) diffeomorphism in the smooth $S^{3}$ case, the equivariant connected sum \# is well defined (that is, does not depend on the choice of $x_{j}$ and $\lambda_{j}$ ), is commutative, and associative. This follows from Theorem $1.6(b)$ because the connected sum of the orbit manifolds has the corresponding properties.

Now Theorem $1.6(c)$ in case $x / S^{p}$ is a sphere, is immediate: $S^{2 p+2}$, 4.1 , has 2 fixed points, $S^{p+1} \times S^{p+1}, 4.2$, has 4 fixed points and $(k-1)$ times the equivariant connected sum of $s^{p+1} \times s^{p+1}$, $k=2,3, \ldots$, has $2 k$ fixed points.

REMARK. Antonelli [3] obtained this classification of the manifold $X$ (but not the action) up to oriented homotopy type if $p=1$ and up to homeomorphism if $p=3$ for almost free smooth $G$-manifolds $X$ with $X / G$ a sphere.

4.4. Plumbing + . Let $x_{j}$ be as in 4.3. Let $\lambda_{j}:\left(D^{p+2}, 0\right) \rightarrow\left(N_{j}, y_{j}\right)$ be (smooth) embeddings with image $\lambda_{j} \cap \pi_{j}\left(F_{j}\right)=\emptyset, \lambda_{1}$ orientation preserving, $\lambda_{2}$ orientation reversing. Let $S^{p}$ act on $S^{p} \times D^{p+2}$ by $g(a, y)=(g a, y)$. There are equivariant homeomorphisms (diffeomorphisms). $\Lambda_{j}: S^{p} \times D^{p+2} \approx \pi_{j}^{-1}$ (image $\lambda_{j}$ ) because, 2.4, $X_{j}-F_{j}$ is a locally trivial principal $S^{p}$-bundle over $N_{j}-\pi_{j}\left(F_{j}\right)$. According to the choice of $\lambda_{j}, \Lambda_{1}$ is orientation preserving, and $\Lambda_{2}$ is orientation reversing. Form the identification space $x_{1}+x_{2}$ from $x_{1}-\pi_{1}^{-1}\left(y_{1}\right)$ and $x_{2}-\pi_{2}^{-1}\left(y_{2}\right)$ by identifying $\Lambda_{1}(g, t u)$ with $\Lambda_{2}(g,(1-t) u)$ for each $u \in s^{p+1}, 0<t<1$. This construction is called plumbing; compare [2, p. 185]. Observe: $X_{1}+X_{2}$ 
is an almost free $S^{p}$-manifold with orbit space $N_{1} \# N_{2}$ (connected sum); the number of fixed points is $v\left(F\left(X_{1}+X_{2}\right)\right)=v\left(F_{1}\right)+v\left(F_{2}\right)$. The same well definedness, and so on, comments apply as for the equivariant connected sum.

REMARK. Due to the uniqueness theorem, Theorem $1.6(b)$, the plumbing $s^{2 p+2}+s^{2 p+2}$ yields $s^{p+1} \times s^{p+1}$ except possibly in the smooth $s^{3}$ case.

4.5. Proof of Theorem $1.6(c)$. Let $N$ be any $(p+2)$-manifold, and let $k$ be any natural number $1,2,3, \ldots$. Let $S^{p}$ act on $S^{p} \times N$ by $g(a, y)=(g a, y)$. According to 4.3 , there is an almost free $s^{p}$-manifold $Y$ with $2 k$ fixed points and orbit space $s^{p+2}$. Then plumbing yields the almost free $S^{p}$-manifold $\left(S^{p} \times N\right)+Y$ with orbit space $N$ and $2 k$ fixed points .

REMARK. Let $Z$ be any principal $S^{2}$-bundle over $N$. Then $z+Y \approx\left(s^{p} \times N\right)+y$ because of the uniqueness theorem, Theorem $1.6(b)$.

4.6. The $s^{3}$-manifold $\Sigma^{8}$. The authors were unable to settle the question whether two almost free smooth $s^{3}$-manifolds with the same number of fixed points and diffeomorphic orbit manifolds are equivariantly diffeomorphic; compare Theorem $1.6(b)$, and Remark 2.10. Thus the following $S^{3}$-manifold may be interesting. Let $\pi: S^{7} \rightarrow S^{4}$ be the Hopf bundle as in 2.1, case $p=3$. Let $\psi: S^{4}+S^{3}$ be the $\pi$ of 4.1 for $p=1$. This is the suspension of the Hopf map $S^{3} \rightarrow S^{2}$ and hence represents the nontrivial element of the homotopy group $\pi_{4}\left(s^{3}\right),[10, \mathrm{p}$. 328]. Define the equivariant diffeomorphism $h: s^{7}+s^{7}$ by $h(z, w)=(z, w) \cdot \psi(\pi(z, w))$ (scalar multiplication from right). Identify the boundaries of $+D^{8}$ and $-D^{8}$ by $h$ to define an almost free $S^{3}$-manifold $\Sigma^{8}$ with two fixed points and orbit space $S^{5}$. According to Theorem $1.6(b), \Sigma^{8}$ is equivariantly homeomorphic to $s^{8}, 4.1$. 
QUESTION. Is $\Sigma^{8}$ equivariantly diffeomorphic to $S^{8}$ ?

\section{References}

[1] J. Frank Adams, Lectures on Lie groups (Benjamin, New York, Amsterdam, 1969).

[2] Peter L. Antonelli, "Structure theory for Montgomery-Samelson fiberings between manifolds. II", Canad. J. Math. 21 (1969), 180-186.

[3] Peter L. Antonelli, "Differentiable Montgomery-Samelson fiberings with finite singular sets", Canad. J. Math. 21 (1969), 1489-1495.

[4] A. Borel, "Fixed point theorems for elementary commutative groups I", Seminar on transformation groups, 157-172 (Annals of Mathematics Studies, 46. Princeton University Press, Princeton, New Jersey, 1960).

[5] William Browder, "Higher torsion in H-spaces", Trans. Amer. Math. Soc. 108 (1963), 353-375.

[6] Morton Brown and Herman Gluck, "Stable structures on manifolds: II. Stable manifolds", Ann. of Math. (2) 79 (1964), 18-44.

[7] Jean Cerf, Sur les difféomorphismes de la sphère de dimension trois $\left(\Gamma_{4}=0\right)$, (Lecture Notes in Mathematics, 53. Springer-Verlag, Berlin, Heidelberg, New York, 1968).

[8] P.T. Church and J.G. Timourian, "Fiber bundles with singularities", J. Math. Mech. 18 (1968), 71-90.

[9] Albrecht Dold, "Fixed point index and fixed point theorem for Euclidean neighborhood retracts", Topology 4 (1965), 1-8.

[10] Sze-Tsen Hu, Homotopy theory (Academic Press, New York, London, 1959).

[11] Dale Husemoller, Fibre bundles (McGraw-Hill, New York, London, Sydney, 1966). 
[12] Klaus Jänich, Differenzierbare G-Mannigfaltigkeiten (Lecture Notes in Mathematics, 59. Springer-Verlag, Berlin, Heidelberg, New York, 1968.

[13] Robion C. Kirby, Laurence C. Siebenmann and Charles T.C. Wall, "The annulus conjecture and triangulation", Notices Amer. Math. Soc. 16 (1969), 432.

[14] John Milnor, Singular points of complex hypersurfaces (Annals of Mathematics Studies, 61. Princeton University Press and University of Tokyo Press, Princeton, New Jersey, 1968).

[15] J. Munkres, "Obstructions to the smoothing of piecewise-differentiable homeomorphisms", Ann. of Math. (2) 72 (1960), 521-554.

[16] M.A. Naimark, Linear representations of the Lorentz group (translated by Ann Swinfen and O.J. Marstrand. Pergamon Press, Oxford; The Macmillan Co., New York, 1964).

[17] Richard S. Palais, "Extending diffeomorphisms", Proc. Amer. Math. Soc. 11 (1960), 274-277.

[18] Edwin H. Spanier, Algebraic topology (McGraw-Hill, New York, San Francisco, St Louis, Toronto, London, Sydney, 1966).

[19] Norman Steenrod, The topology of fibre bundles (Princeton Mathematical Series, 14. Princeton University Press, Princeton, New Jersey, 1951).

[20] J.G. Timourian, "Fiber bundles with discrete singular set", J. Math. Mech. 18 (1968), 61-70.

Department of Mathematics,

Syracuse University,

Syracuse,

New York, USA;

Mathematisches Institut der Universitat Köln, Köln,

Germany. 